\title{
LUDICIDADE APLICADA AO ENSINO DO CORPO HUMANO: ORIENTAÇÃO E DIVULGAÇÃO DOS MÉTODOS CONTRACEPTIVOS PARA PREVENÇÃO DA GRAVIDEZ PRECOCE E DOENCAS SEXUALMENTE TRANSMISSÍVEIS EM ESCOLARES AMAZÔNICOS
}

\author{
AYRTON BATISTA RODRIGUES \\ ROSIELY SILVA CABÚS \\ PROF. ${ }^{A}$ DR. ${ }^{A}$ SILVIA REGINA SAMPAIO FREITAS
}

\begin{abstract}
RESUMO
A ludicidade possui um papel significativo para o ensino de Ciências e Biologia. Inserido nesse contexto, o presente estudo apresenta uma sequência didática pautada no uso de jogos didáticos como metodologia complementar à orientação e divulgação dos métodos contraceptivos para prevenção da gravidez precoce e de doenças sexualmente transmissíveis. Este estudo foi idealizado e executado no segundo semestre de 2016, em uma escola pública localizada na área urbana de Tefé/AM. O público-alvo do estudo foram 58 alunos (38 meninas e 20 meninos) do oitavo ano do Ensino Fundamental. Os estudantes que participaram do estudo tinham entre 13 a 18 anos de idade, na época da execução da atividade. A sequência didática foi estruturada em quatro encontros pedagógicos: $1^{\circ}$. encontro: avaliação do conhecimento prévio por meio de questionário (pré-teste); do $2^{\circ}$. ao $3^{\circ}$. encontro: problematização do tema; $4^{\circ}$. encontro: avaliação do conhecimento adquirido por intermédio de jogo didático e questionário (pós-teste). A análise dos resultados do questionário pós-teste permitiu verificar que os alunos construíram novos conhecimentos com a atividade lúdica desenvolvida com o jogo didático. Comparando os resultados obtidos no pré e pós-teste, foi possível perceber que os alunos responderam às questões de forma bem elaborada e correta após a utilização do jogo didático. Os resultados obtidos neste estudo permitiram verificar que os alunos acreditam que essa atividade lúdica é uma ótima estratégia
\end{abstract}


de ensino, pois facilitou a compreensão e fixação dos conceitos transmitidos nas aulas teóricas.

Palavras-chave: Jogos Didáticos. Ciências. Ensino Fundamental.

\begin{abstract}
Lududicity plays a significant role in science and biology. In this context, the present study presents a didactic sequence based on the use of games as an alternative method to the orientation and dissemination of contraceptive methods for the prevention of early pregnancy and sexually transmitted diseases. This study was executed in 2016, in a public school located in the urban area of Tefé /AM. The methodology was tested and evaluated by fifty-eight students (thirty-eight girls and twenty boys) from a public school in Tefé, Amazonas. Students who participated in the study were between the ages of 13 and 18 at the time of the activity. The didactic sequence was structured in four pedagogical meetings: 1st meeting: evaluation of previous knowledge through questionnaire (pre-test); from the 2nd to the 3rd meeting: problematization of the theme; 4th meeting: evaluation of knowledge acquired through didactic game and questionnaire (post-test). The analysis of the results of the post-test questionnaire allowed us to verify that the students constructed new knowledge with the play activity developed with the didactic game. Comparing the results obtained in the pre- and post-test, it was possible to perceive that the students answered the questions in a very elaborate and correct way after the use of the didactic game. The results obtained in this study allowed to verify that the students believe that this play activity is an excellent teaching strategy, because it facilitated the understanding and fixation of the concepts transmitted in the theoretical classes.
\end{abstract}

Key-words: Didactic Games. Sciences. Elementary School.

\title{
INTRODUÇÃO
}

O ensino de Ciências Naturais destaca-se como um espaço em que os fenômenos da natureza e as transformações produzidas pelo homem podem ser investigados, possibilitando que 
os estudantes compreendam a natureza e o ser humano como parte integrante e agente de transformações do mundo em que vive (KAWAMOTO et al., 2014). No decorrer do ensino fundamental, é proposto o desenvolvimento de diferentes eixos temáticos, entre eles "Ser Humano e Saúde" (BRASIL, 2000).

O ensino de temas relacionados ao funcionamento do corpo humano e os aspectos de saúde almejam que os alunos desenvolvam juízo crítico e capacidade de intervenção sobre suas vidas e acerca do ambiente com o qual interagem (BRASIL, 2000). Para tanto, faz-se necessário que o estudante conheça o seu próprio corpo e possa cuidar-se, valorizando e adotando hábitos saudáveis, e agindo com responsabilidade em relação à sua saúde e à saúde coletiva (RABELO, 1994; KAWAMOTO et al., 2014). Inserido no eixo temático Ser Humano e Saúde, encontra-se o assunto Reprodução Humana. Tal assunto visa aprofundar o conhecimento a respeito dos aspectos anatômicos e funcionais dos sistemas reprodutores feminino e masculino, o mecanismo de reprodução da espécie humana, bem como, as possibilidades de doenças que podem ser transmitidas por intermédio do ato sexual (BRASIL, 2000). Segundo os parâmetros Curriculares Nacionais para o ensino fundamental, é sugerido que as aulas relacionadas com o assunto Reprodução Humana sejam complementadas por atividades lúdicas, a fim de tornar o processo de ensino e aprendizagem efetivo (BRASIL, 2000).

As atividades lúdicas envolvem uma diversidade de recursos didáticos que são utilizados como suporte experimental na organização do processo de ensino e da aprendizagem, com a finalidade de servir de interface mediadora para facilitar na relação entre professor, aluno e o conhecimento, em um momento preciso da elaboração do saber (KAWAMOTO et al., 2014). Pode-se compreender que os recursos didáticos têm sido concebidos como "instrumentos modernizadores das práticas escolares e, consequentemente, efetivadores de um ensino efetivo e de qualidade" (KAWAMOTO et al., 2014). Entre as opções de recursos didáticos disponíveis para os professores de Ensino Fundamental, estão os jogos didáticos. O ensino por meio de jogos possibilita a criação de um ambiente motivador, o que 
facilita a dinâmica do processamento cognitivo de informações. Isto implica ainda na criação de um espaço pedagógico e dialógico que favoreça a construção contínua de processos internos simples de estruturação de realidades (ZUANON et al., 2010).

Inserido nesse contexto, o presente estudo apresenta uma sequência didática pautada no uso de jogos didáticos como metodologia complementar à orientação e divulgação dos métodos contraceptivos para prevenção da gravidez precoce e de doenças sexualmente transmissíveis.

\section{PROCEDIMENTO METODOLÓGICO}

Este estudo foi idealizado e executado no segundo semestre de 2016, em uma escola pública situada na área urbana de Tefé/ AM. Esse município encontra-se localizado no interior do Estado do Amazonas, distante $574 \mathrm{~km}$ da capital Manaus, às margens do Rio Solimões (IBGE, 2010).

Tefé é um município-polo da região do Triângulo Jutaí-Solimões-Juruá, com o maior número de instituições educacionais da região, possuindo universidade, instituto federal de ensino técnico e grande rede de escolas de ensino público e particular. A rede de ensino do município possui 108 estabelecimentos escolares, sendo 106 públicos e dois particulares. Desse total, sete estão situadas na área rural e 101 na área urbana de Tefé (IBGE, 2010).

A escola envolvida neste estudo é uma das instituições participantes do Programa Institucional de Bolsas de Iniciação à Docência (PIBID), que em parceria com o Centro de Estudos Superiores de Tefé - Universidade do Estado do Amazonas desenvolve projetos de ensino e pesquisa voltados para o aperfeiçoamento das práticas pedagógicas.

\section{Sujeitos da Pesquisa e os Critérios para Seleção dos Participantes}

O público-alvo do presente estudo foram 58 alunos (38 meninas e 20 meninos) de duas turmas de oitavo ano do Ensino Fundamental. Os estudantes que participaram do estudo tinham entre 13 a 18 anos de idade, na época da execução da atividade. 
Os critérios para inclusão dos participantes no estudo foram: ser aluno do oitavo ano do ensino fundamental, e ter interesse em participar das atividades pedagógicas desenvolvidas no Subprojeto do PIBID-Biologia. Excluiu-se da pesquisa os alunos ausentes no dia da execução das atividades pedagógicas.

Todas as atividades conduzidas na sala de aula foram acompanhadas pelo professor-supervisor do Subprojeto do PIBID-Biologia e também responsável da disciplina "Ciências".

\section{Descrição da Sequência Didática}

A sequência didática "Ludicidade como metodologia complementar ao ensino do Corpo Humano: orientação e divulgação dos métodos contraceptivos para prevenção da gravidez precoce e de doenças sexualmente transmissíveis" foi organizada em quatro etapas pedagógicas distintas (adaptada de TERÇO e FREITAS, 2016): a) Conhecimento Prévio, b) Problematização do Assunto, c) Aplicação do Conhecimento Adquirido, e d) Satisfação com a Atividade. A sequência didática foi desenvolvida em quatro aulas sucessivas, com duração de 45 minutos cada. A seguir, o resumo das atividades propostas para a realização da sequência didática.

Conhecimento Prévio: a primeira etapa teve duração de uma aula e visou avaliar o conhecimento prévio dos alunos sobre o tema "métodos contraceptivos para prevenção da gravidez precoce e de doenças sexualmente transmissíveis". Para tal, os alunos do oitavo ano do ensino fundamental foram convidados a responder a um pré-teste com três perguntas sobre o tema. As questões utilizadas foram: (1) O que é gravidez precoce?; (2) O que significa "método contraceptivo"?; (3) Cite três tipos de métodos contraceptivos. As respostas obtidas no questionário para avaliação do conhecimento prévio foram categorizadas em Resposta Correta e Resposta Incorreta. Para a análise qualitativa do questionário utilizamos a Análise de Conteúdo, como proposto por Bardin (2011). Os resultados foram tabulados em planilha de Excel $^{\circledR}$ e apresentados em forma de gráfico de barras.

Problematização do Assunto: esta etapa teve duração de duas aulas consecutivas. O conteúdo teórico foi apresentado de 
forma dialogada e com auxílio de quadro branco e data show, e de manequim do corpo humano. $\mathrm{Na}$ aula seguinte, os alunos conversaram com uma profissional de saúde sobre assuntos relacionados ao tema.

Aplicação do Conhecimento Adquirido: para avaliar o conhecimento adquirido com a atividade, empregou-se a estratégia do jogo didático. Foram elaborados dois tipos de jogo, um intitulado "jogo da memória - métodos contraceptivos (Figura 1)", e o segundo "jogo da verdade sobre sexualidade". Essa etapa teve duração de uma aula.

Figura 1: Jogo da memória sobre os métodos contraceptivos

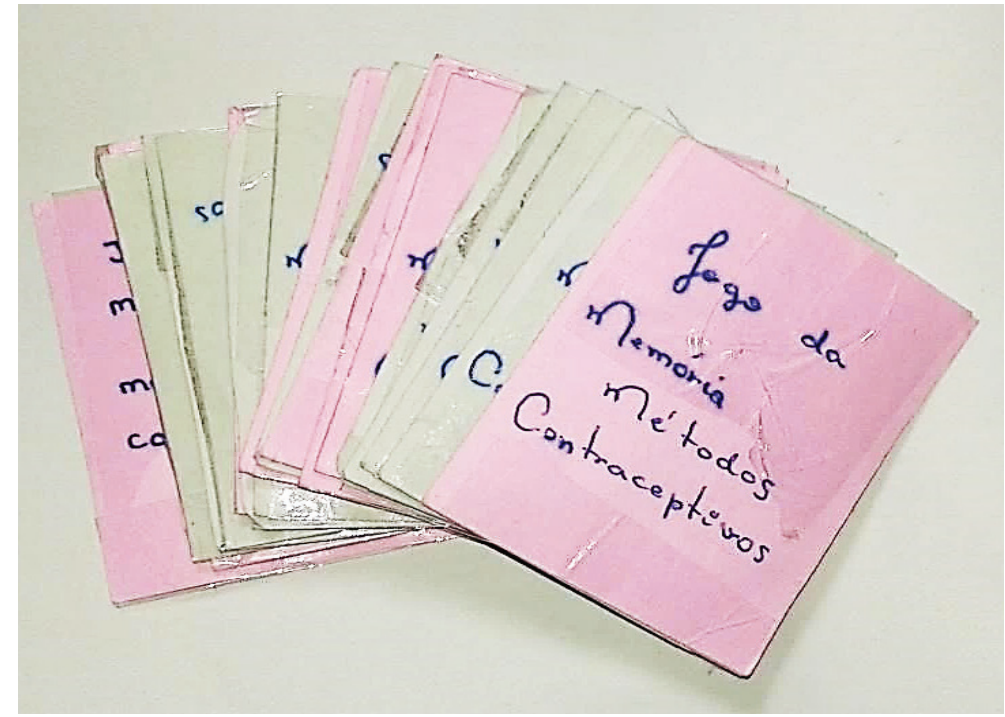

A primeira atividade foi inspirada nos princípios do jogo da memória. Foram confeccionados 10 pares de cartas utilizando material de baixo custo, como: (a) Duas cartolinas, uma na cor rosa e outra de cor bege para confecção de 20 cartas, cada uma na dimensão de $10 \mathrm{~cm}$ de altura por $6 \mathrm{~cm}$ de largura; (b) Caneta esferográfica azul; (c) Tesoura sem ponta; (d) Régua de $15 \mathrm{~cm}$; (e) Informações e fotos relacionados ao tema; e (f) Fita adesiva transparente ou cola branca - para fixar as figuras na base de 
cartolina. Cada par de cartas continha informações complementares (p. ex.: definição/descrição e imagem correspondente) sobre a temática da sequência didática. A dinâmica do jogo ocorreu da seguinte forma: o aluno escolhia uma carta; depois tinha que encontrar a carta com as informações complementares. $\mathrm{O}$ aluno foi estimulado a opinar se as cartas escolhidas continham informações complementares. No caso das informações de uma carta não serem complementares com a da outra, as cartas então, voltavam para o tabuleiro do jogo.

Para a construção do "jogo da verdade sobre a sexualidade" (Figura 2) também utilizou-se material de baixo custo como garrafa pet de 2L; duas folhas de papel crepom, sendo uma na cor azul e a outra na cor vermelha; imagens dos símbolos masculino (ङ) e feminino (9); dez cartas contendo frases sobre gravidez precoce, métodos contraceptivos e doenças sexualmente transmissíveis. As cartas foram confeccionadas em papel cartolina rosa e azul, um processo similar ao descrito no jogo da memória.

Figura 2: Jogo da verdade sobre a sexualidade

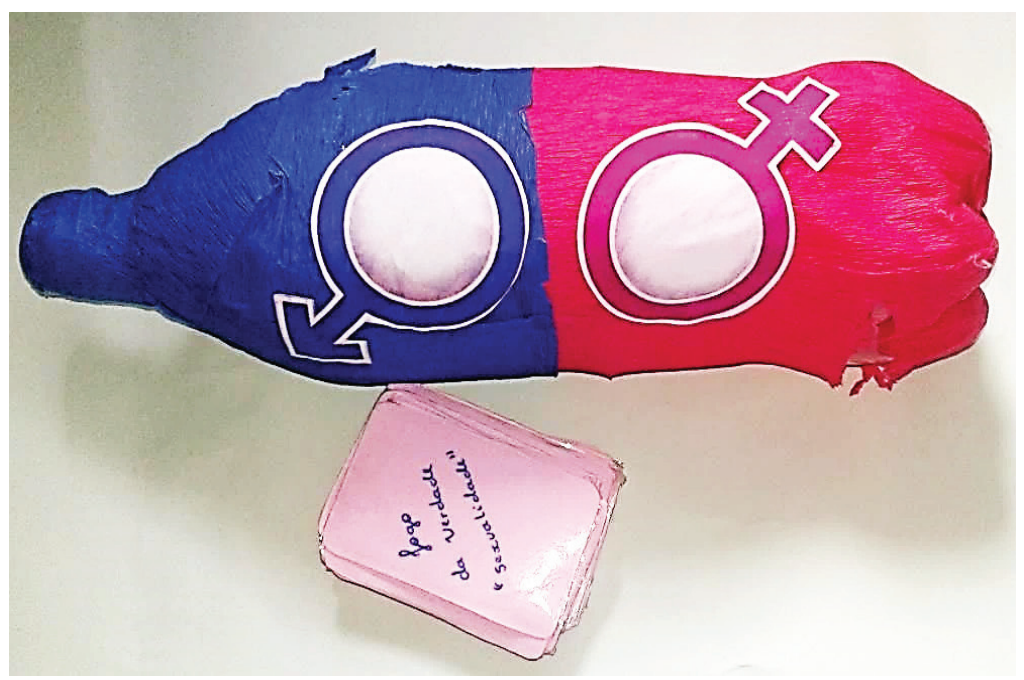


As regras dos jogos incluíram: formar um grupo com até cinco participantes, que por sorteio definiram a ordem de jogada de cada um. O participante da vez rodava a garrafa em sentido horário. Ao parar de rodar, a ponta da garrafa (extremidade com a tampa da garrafa) indicava o jogador que deveria tirar uma carta do baralho. O texto presente na carta era lido em voz alta, e o participante deveria dizer se a informação era verdadeira ou falsa.

Satisfação com a Atividade: No último dia da sequência didática, os alunos foram reavaliados por meio de questionário. As questões presentes no questionário utilizado na avaliação prévia do conhecimento foram reapresentadas para detectar se houve mudanças em relação às opiniões iniciais. Adicionalmente, incluiu-se duas perguntas para avaliar o grau de satisfação dos alunos quanto ao uso dos jogos didáticos na aula de Ciências.

\section{RESULTADOS E DISCUSSÃO}

Conhecimento Prévio dos Métodos Contraceptivos para Prevenção da Gravidez Precoce e de Doenças Sexualmente Transmissíveis

O início da sequência didática "Ludicidade como metodologia complementar ao ensino do Corpo Humano: orientação e divulgação dos métodos contraceptivos para prevenção da gravidez precoce e de doenças sexualmente transmissíveis" foi marcado pela análise do conhecimento prévio dos alunos sobre o tema. Para tanto, os alunos responderam a um questionário com três questões dissertativas. Os resultados obtidos pela análise do conteúdo estão sumarizados no Gráfico 1.

A partir da avaliação exploratória inicial verificou-se que os alunos não tinham conhecimento consolidado sobre os assuntos de gravidez precoce, métodos contraceptivos e doenças sexualmente transmissíveis. Quando foi aplicado o questionário pré-teste, alguns alunos disseram "Professora eu não sei responder essas perguntas". Apesar dessa negativa inicial, os alunos foram incentivados a responder as questões considerando a realidade do aluno. O índice de acerto variou de $24 \%$ a $50 \%$. 
Gráfico 1: Percentual de respostas corretas (barras escuras) e incorretas (barras claras) obtidas na avaliação do conhecimento prévio dos alunos sobre o tema "gravidez precoce e métodos contraceptivos".

O que é gravidez precoce?

$\mathrm{O}$ que significa método contraceptivo?

Cite três tipos de métodos contraceptivos.

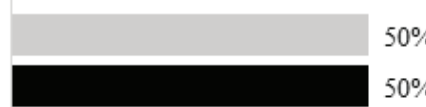

$50 \%$
$76 \%$

$24 \%$

$74 \%$

$26 \%$

\section{$\%$ das respostas}

Apesar do rendimento insatisfatório na avaliação prévia, observamos que durante a apresentação teórica dos assuntos relacionados com a gravidez precoce, métodos contraceptivos e doenças transmitidas sexualmente, os alunos mantiveram atenção e foco. De forma expositiva, foram exibidos os métodos contraceptivos existentes, bem como, o sistema reprodutor de ambos os gêneros. Com o auxílio de manequins do corpo humano, foi possível demonstrar a forma correta do uso do preservativo masculino e da camisinha feminina. Também discutiu-se a importância de vacinação contra o vírus Papiloma Humano - HPV, que faz parte do calendário nacional de vacinação. Por fim, houve distribuição de preservativos e de panfletos informativos.

Inicialmente, os alunos mostraram-se tímidos ao participar da aula, porém, superado seu acanhamento inicial, interagiram, esclareceram muitas dúvidas e, sem inibição, receberam as camisinhas distribuídas. De fato, a escola é o ambiente ideal para o desenvolvimento de estratégias de conscientização e de promoção da saúde dos jovens e adolescentes, uma vez que atitudes e valores são formados nesse ambiente (SANTOS et al., 2012; OLIVEIRA et al., 2013; GONÇALVES et al., 2016). Também 
é importante ressaltar que o trabalho em educação sexual com adolescentes não deve ser visto como uma domesticação dos indivíduos, mas como um processo de autorreflexão, fazendo que eles possam se estabelecer como sujeitos, além de exercer uma visão crítica diante da sexualidade, de modo a contribuir com os ideais emancipatórios da humanidade e garantir o respeito ao próximo e às diferentes formas de exercício da sexualidade (GONÇALVES et al., 2016).

\section{A Ludicidade como Estratégia Complementar ao Ensino do Corpo Humano}

A incorporação de atividades lúdicas, como jogo da memória e o jogo da verdade sobre a sexualidade, mostrou-se capaz de despertar a curiosidade e o interesse dos alunos em compreender os conceitos inerentes ao tema "Ludicidade como metodologia complementar ao ensino do Corpo Humano: orientação e divulgação dos métodos contraceptivos para prevenção da gravidez precoce e de doenças sexualmente transmissíveis". No curso das atividades, verificou-se um intenso debate entre os alunos, gerando assim um diálogo intenso e produtivo. É válido ressaltar que no dia previsto para a realização dessa atividade nenhum aluno faltou ou chegou atrasado.

Esse comportamento corrobora os relatos de Almeida e colaboradores (2015) e Silva e colaboradores (2016). Segundo esses autores, o uso de sequências didáticas inovadoras e pautadas no uso de atividades lúdicas contribui significativamente para o aumento da frequência escolar, e por conseguinte do rendimento acadêmico. Acredita-se que funções cognitivas como motricidade, linguagem, percepção, representação, memória estão profundamente interligadas, e por isso são estimuladas pelo ato de "brincar" (KAWAMOTO et al., 2014; GONÇALVES et al., 2016). Corroborando essa ideia, outros estudos indicaram que o lúdico não se limita ao entretenimento, mas constitui uma ferramenta pedagógica que facilita a aquisição do conhecimento sobre Ciências Naturais pelos alunos (SOUZA e FREITAS, 2016).

A ludicidade não pode ser vista como diversão, uma vez que facilita a aprendizagem e o desenvolvimento interpessoal, 
possibilitando ao aluno aprender de modo descontraído e atuando na melhoria da saúde, o que facilita os processos de socialização, comunicação, expressão e construção do conhecimento (KAWAMOTO et al., 2014; GONÇALVES et al., 2016).

\section{Avaliação do Conhecimento Adquirido}

Os resultados do questionário para avaliação do conhecimento adquirido estão apresentados no Gráfico 2. Com base na análise comparativa dos resultados obtidos na avaliação prévia e adquirida, ficou evidente a eficácia da sequência didática pautada no uso de técnicas de ensino tradicional e lúdica para abordar temas sobre gravidez precoce e métodos contraceptivos. O índice de respostas corretas na segunda rodada de avaliação ficou acima dos $90 \%$ para todas as questões.

Gráfico 2: Percentual de respostas corretas (barras escuras) e incorretas (barras claras) obtidas na avaliação do conhecimento adquirido dos alunos sobre o tema "gravidez precoce e métodos contraceptivos".

O que é gravidez precoce?

$\mathrm{O}$ que significa método contraceptivo?

Cite três tipos de métodos contraceptivos.

\section{$2 \%$}

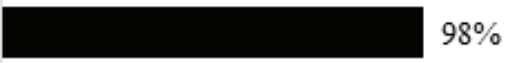

$10 \%$

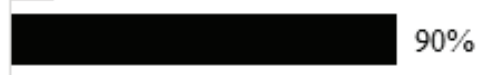

$2 \%$

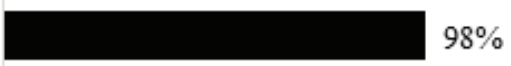

$\%$ das respostas 
A partir dos resultados obtidos, percebe-se que inserir metodologias alternativas nas aulas de Ciências auxilia a viabilizar uma forma mais atrativa de ensinar os conteúdos, pois os alunos são receptivos a esse tipo de prática. De fato, diversos estudos já evidenciaram a boa receptividade das metodologias alternativas de ensino pelos alunos (OLIVEIRA et al., 2015, SILVA et al., 2016), bem como, a eficácia dessas abordagens na complementação do ensino convencional (MERÇON, 2015). No entanto, é essencial que o docente faça uso de questões do cotidiano para subsidiar questionamentos que estimulem a compreensão dos conceitos teóricos apresentados (SILVA-FILHA et al., 2016). Os resultados obtidos neste estudo, em consonância com as diretrizes exibidas pelos Parâmetros Curriculares Nacionais, apontam a importância de selecionar e integrar estratégias diversificadas que possam promover um aprendizado significativo no âmbito do ensino de ciências e da promoção da saúde (BRASIL, 2000).

\section{Resultados da Pesquisa de Satisfação}

As duas últimas perguntas do questionário serviram para dimensionar a opinião dos alunos sobre a atividade. Essa pesquisa de opinião revelou que os recursos didáticos foram considerados válidos para auxiliar no entendimento a respeito dos métodos contraceptivos para prevenção da gravidez precoce e de doenças sexualmente transmissíveis.

Quando questionados sobre "A utilização de métodos lúdicos (jogos) ajudou a despertar a curiosidade e o interesse pelo assunto?", 100\% dos alunos concordaram com essa afirmativa. Corroborando os nossos resultados, os estudos ancorados no uso de alternativas pedagógicas, como jogos de cartas (FARIAS et al., 2015), gibis (CARLAN et al., 2013), dobraduras (CONDE et al., 2013), protótipos anatômicos (SILVA-FILHA et al., 2016) e modelos didáticos (SILVA et al., 2017) também mostraram a eficiência da metodologia na promoção do interesse dos alunos de forma que desenvolvam o raciocínio, a imaginação, a memória e a curiosidade.

Ao serem questionados sobre "A metodologia de ensino facilitou a aprendizagem?” (Gráfico 2), 100\% dos alunos concor- 
daram que as atividades lúdicas (jogos didáticos) promoveram a aprendizagem de forma construtiva. Esses resultados superam os descritos por Sant'anna e colaboradores (2011), que verificaram a opinião de discentes ao realizarem um jogo didático de tabuleiro e constataram que $83 \%$ dos participantes acreditam ser importante o uso de jogos em sala de aula. Segundo os autores, $85 \%$ dos alunos responderam que o dinamismo do jogo contribuiu para a aprendizagem do conteúdo, bem como, $87 \%$ dos estudantes afirmaram que o jogo auxiliou na aprendizagem de conceitos não conhecidos.

\section{CONSIDERAÇÕES FINAIS}

Com uma sequência didática pautada em problematização do tema associado a atividades lúdicas (jogo da memória e jogo da verdade sobre a sexualidade), foi possível conscientizar e orientar os alunos, de forma divertida e prazerosa, a respeito da gravidez precoce e a importância do uso dos métodos contraceptivos.

\section{FONTES DE FINANCIAMENTO}

O presente estudo foi financiado pela CAPES, por meio do Programa Institucional de Bolsas a Iniciação à Docência (PIBID).

\section{REFERÊNCIAS}

ALMEIDA, C. M. M.; LOPES, L. A.; LOPES, P. T. C. Sequências didáticas eletrônicas no ensino do corpo humano: comparando o rendimento do ensino tradicional com o ensino utilizando ferramentas tecnológicas. Acta Scientiae, v. 17, n. 2, p. 466-482, 2015.

BARDIN, L. Análise de conteúdo. São Paulo: Edições 70, 2011, 80p.

BRASIL. Secretaria de Educação Fundamental. Parâmetros curriculares nacionais: Ciências Naturais/Secretaria de Educação Fundamental. Brasília: $\mathrm{MEC} / \mathrm{SEF}, 2000$, 138p.

BORUCHOVITCH, E.; BZUNECK, A. J. Aprendizagem: processos psicológicos e o contexto social na escola. Petrópolis: Editora Vozes, 2004.

CARLAN, F. A.; SEPEL, L. M. N.; LORETO, E. L. S. Explorando diferentes recursos didáticos no Ensino Fundamental: uma proposta para o ensino de célula. Acta Scientiae, v. 15, n. 2, p. 338-353, 2013.

CONDE, T. T.; MENDES, L. L.; BAY, M. Utilização de metodologias al- 
ternativas na formação dos professores de biologia no IFRO - CAMPUS ARIQUEMES. Revista Labirinto, v. 13, n. 18, p. 139-147, 2013.

FARIAS, L. F.; SILVEIRA, G. F.; ARRUDA, V. M. O jogo do ciclo celular uma alternativa para o ensino de biologia. Revista Amazônica de Ensino de Ciências -ARETÉ, v. 8, n. 16, p. 27-35, 2015.

GONÇALVES, L. F. F.; FARIA, D. S. A.; BATISTA, E. S.; FERREIRA, S. R.; ASSIS, S. M. Promoção de saúde com adolescentes em ambiente escolar: relato de experiência. SANARE, v. 15 n. 2, p. 160-167, 2016.

IBGE. Instituto Brasileiro de Geografia e Estatística [Internet]. Censo 2010: Cidades. [Acesso em 2013.] Disponível em: <http://www.cidades.ibge.gov.br/ painel $/$ populacao.php?lang=\&codmun $=130420 \&$ search $=\% 7$ Ctefe $>$.

KAWAMOTO, E. M.; CAMPOS, L. M. Histórias em quadrinhos como recurso didático para o ensino do corpo humano em anos iniciais do ensino fundamental. Ciência \& Educação, v. 20, n. 1, p. 147-158, 2014.

MERÇON, F. Os objetivos das ciências naturais no ensino médio. Revista Eletrônica do Vestibular, v. 22, n. 8, p. 38, 2015.

OLIVEIRA, D. B.; PIANCA, B. R.; SANTOS, E. E. R.; MANCINI, K. C. Modelos e atividades dinâmicas como facilitadores para o ensino de biologia. Enciclopédia Biosfera, v. 11, n. 20, p. 514-514, 2015.

OLIVEIRA, K. N. S.; BEZERRA, M. A. R.; ROCHA, R. C.; SANTOS, L. R.; SARAIVA, P. V. S. Educação sexual na adolescência e juventude: abordando as implicações da sexualidade no contexto escolar. Sanare, v. 12, n. 2, p. 7-13, 2013.

RABELLO, S. H. S. A criança, seu corpo, suas idéias. Ensino em Re-Vista, v. 3, n. 1, p. 15-29, 1994.

SANT'ANNA, I. C.; BRANCO, A. L. C.; PEREIRA, K. P.; CARVALHO, A. C. P.; TAVARES, M. G. Perfil da Genética: Uma maneira divertida de memorizar conteúdos. Genética na Escola, v. 6, n. 2, p. 17-29, 2011.

SANTOS, K. T.; GARBIN, A. J. I.; GARBIN, C. A. S. Saúde bucal nas escolas: relato de experiência. Revista Ciência em Extensão, v. 8, n. 1, p. 16-19, 2012. SILVA, A. A.; SILVA-FILHA, T. T.; FREITAS, S. R. S. Utilização de modelo didático como metodologia complementar ao ensino da anatomia celular. Biota Amazônia, v. 6, n. 3, p. 17-21, 2016.

SILVA-FILHA, R. T.; SILVA, A. A.; FREITAS, S. R. S. Uma alternativa didática às aulas tradicionais de ciências: aprendizagem colaborativa e modelização aplicadas ao ensino do sistema urinário. Cadernos de Educação, v. 15, n. 31, p. 87-105, 2016.

SOUZA, L. L.; FREITAS, S. R. S. O ensino de Ciências e Biologia no Amazonas: experiências do PIBID no município de Tefé. Jundiaí: Paco Editorial, 2016, 136p.

TERÇO, J. S., FREITAS, S. R. S. Educação e saúde na escola: aplicação de uma sequência didática sobre alimentação saudável. Cadernos de Educação, v. 15, n. 31, p. $71-85,2016$. 
ZUANON, A. C. A.; DINIZ, R. H. S.; NASCIMENTO, L. H. Construção de jogos didáticos para o ensino de Biologia: um recurso para integração dos alunos à prática docente. Revista Brasileira de Ensino de Ciência e Tecnologia, v. 3, n. 3, p. 49-59, 2010.

\section{SOBRE OS AUTORES}

Ayrton Batista Rodrigues é graduado em Ciências Biológicas - Licenciatura. Centro de Estudos Superiores de Tefé. Universidade do Estado do Amazonas. E-mail: ayrtonbatista19@gmail.com

Rosiely Silva Cabús é professora da Escola Municipal Professor Helyon de Oliveira e Supervisora do Subprojeto do PIBID em Biologia.

E-mail: rosadejahsubac@gmail.com

Silvia Regina Sampaio Freitas é professora adjunta do Curso de Licenciatura em Ciências Biológicas. Centro de Estudos Superiores de Tefé. Universidade do Estado do Amazonas (CEST-UEA). Coordenadora do Subprojeto do Programa Institucional de Bolsa de Iniciação à Docência e do curso de Licenciatura em Ciências Biológicas - Oferta Especial. Possui Mestrado e Doutorado em Genética Humana pelo Instituto Oswaldo Cruz - FIOCRUZ e Pós-Doutorado pela Faculdade de Medicina da USP.

E-mail: silvia.sampaio.freitas@hotmail.com 